\title{
Evaluating Machine Creativity
}

\author{
Anna Jordanous \\ Music Informatics / Creative Systems Lab \\ Department of Informatics, University of Sussex, UK \\ a.k.jordanous@sussex.ac.uk
}

\begin{abstract}
Can a computer be creative? And what can we learn about our own creativity from studying computational creativity? My research offers a comprehensive and practically useful investigation into how to evaluate the level of creativity demonstrated by computational systems. How should something so subjective as creativity be measured? I argue that it is most productive to treat creativity as a collection of inter-related factors such as originality, value and productivity, which are more tightly defined and therefore more amenable to measurement. Potential factors are being derived from empirical studies examining a wide variety of our writings on creativity. These will be tested in a simulation of a creative environment: the best performing factors will be applied to evaluation of existing creative systems, in comparison to assessments made by human judges. The aim of this is to identify key components for creativity, giving insight into how to approach the evaluation and improvement of computational systems and also towards human creativity as well.
\end{abstract}

\section{Author Keywords}

Creativity, Evaluation, Aspects of Creativity

\section{ACM Classification Keywords}

I.2.0 Artificial Intelligence: General-Cognitive simulation

\section{General Terms}

Experimentation, Measurement, Standardization

\section{EXAMINING HOW WE PERCEIVE CREATIVITY}

The primary resource we have for examining creative actions is that of ourselves; how humans demonstrate creativity. The closer artificial creative systems can match to our perception of human creativity, the more successful they are generally deemed to be for demonstrating creativity. Therefore we need to clarify what humans consider to be creative.

I have conducted a number of empirical studies on how we have attempted to capture the nature of creativity in words, in definitions and in papers from a variety of academic viewpoints from psychology [1] to computational creativity [2]. These results (for example see Figure 1) provide the basis for further exploration of key factors in creativity.

Copyright is held by the author/owner(s).

$C \& C^{\prime} 09$, October 26-30, 2009, Berkeley, California, USA.

ACM 978-1-60558-403-4/09/10.

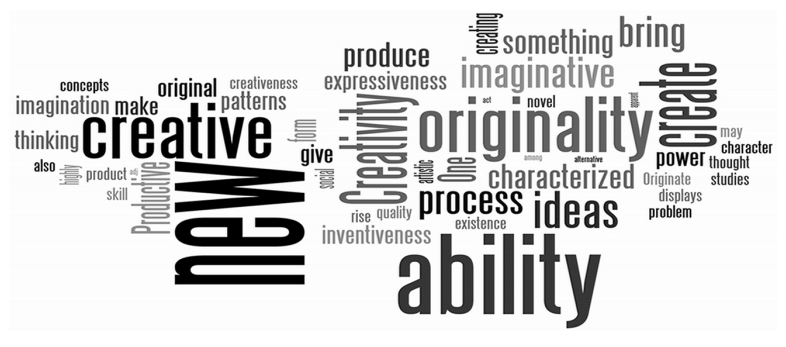

Figure 1. Words appearing frequently in 23 definitions of 'creativity'

The factors identified in my current empirical work will be tested in a simulation of a creative environment emulating several creative producers and their critics interacting with each other over time (in a multi-agent system). Those factors that perform most promisingly in simulation will be tested further in experiments with human participants, and in evaluation of existing creative systems, to find which factors are most important in gauging the level of creativity present.

\section{EXPECTED CONTRIBUTIONS}

The aim of this work is to identify key components of creativity, to enable more detailed, cognitively-based evaluation of our progress in computational creativity. This also gives some insight into the nature of human creativity.

\section{BIOGRAPHY}

Currently a 2nd year doctoral student at the University of Sussex, UK, Anna is a member of the Music Informatics Research Centre and the Creative Systems Lab at Sussex and is supervised by Nick Collins and Chris Thornton. Before starting her doctoral study, Anna obtained an MSc in Artificial Intelligence from the University of Edinburgh (2007) and a BSc in Computer Science from the University of Warwick (2001). Anna has previously published interdisciplinary work combining artificial intelligence and music. She has also presented work reflecting upon parallels between linguistic and musical creativity. Her doctoral study examines the question of how to evaluate creativity and compare creative systems on the extent of creativity they exhibit.

\section{REFERENCES}

1. J. P. Guilford. Creativity. American Psychologist, 5:444-454, 1950.

2. G. Ritchie. Some empirical criteria for attributing creativity to a computer program. Minds and Machines, 17:67-99, 2007. 